\title{
Schwann Cells Proliferate at Rat Neuromuscular Junctions during Development and Regeneration
}

\author{
Flora M. Love and Wesley J. Thompson \\ Department of Zoology, University of Texas at Austin, Austin, Texas 78712
}

Terminal Schwann cells (TSCs) cover neuromuscular junctions and are important in the repair and maintenance of these synapses. We have examined how these cells are generated at developing junctions and how their number is regulated during repair of nerve injury. At birth, approximately half of the junctions in rat soleus and extensor digitorum longus muscles have one TSC soma. Somata are absent from the remainder, although Schwann cell (SC) processes arising from somata along the preterminal axon cover almost all of these synapses. By 2 months of age, junctions have gained an additional two to three TSCs. Most of this gain occurs during the first 2 postnatal weeks and largely precedes the expansion of endplate size. Although the initial addition is caused by cell migration, mitotic labeling shows extensive division of TSCs at junctions. A slower addition of TSCs occurs in adult muscles, and TSC number in the adult is correlated with endplate size.
During repair of nerve injury, TSC number is regulated by a combination of signals from motor neurons and denervated tissue. As shown previously (Connor et al., 1987), denervation of adult muscles did not, in itself, cause TSC mitosis. However, TSCs became mitotic during reinnervation. Partial denervation induced division of TSCs at innervated but not denervated endplates. A disproportionate number of these mitotic cells were found at endplates contacted by TSC processes extended from nearby denervated endplates, contacts known to promote nerve sprouting. These results show an association between TSC mitotic activity and alterations in synaptic structure during development, sprouting, and reinnervation.

Key words: Schwann cell; proliferation; mitosis; migration; cell number; neuromuscular junction; development; partial denervation; reinnervation; denervation; sprouting; endplate area
Schwann cells cover the terminal arborizations of motor axons at neuromuscular junctions. These "terminal" Schwann cells (TSCs) are important in the repair of synapses after nerve injury. At the time of muscle denervation, TSCs extend processes that guide regenerating axons (Son and Thompson, 1995a). In partially denervated muscles, TSC processes extend from denervated synaptic sites and grow to nearby innervated synaptic sites. These TSC "bridges" induce nerve terminals to grow or "sprout" and guide these sprouts to the denervated synaptic sites (Son and Thompson, 1995b).

Several observations suggest that TSCs also function in the normal maintenance of neuromuscular junctions. TSCs are known to sense synaptic activity at neuromuscular junctions. These cells possess muscarinic and purinergic receptors that bind acetylcholine and ATP, respectively, released by the nerve terminals (Robitaille, 1995; Robitaille et al., 1997). As a consequence of the second messengers induced by binding of acetylcholine to the muscarinic receptors and the action of these messengers on internal calcium stores, synaptic activity results in an elevation of intracellular calcium in TSCs (Jahromi et al., 1992). These activity-induced calcium fluctuations appear to regulate the expression of certain genes: blockade of neural activity or of transmitter release evokes expression of the gene for glial fibrillary acidic protein, a protein that may be important in TSC

Received May 28, 1998; revised Aug. 25, 1998; accepted Aug. 28, 1998.

This work was supported by grants from National Institutes of Health and the National Science Foundation. We thank Ying Lu for advice on the BrdU protocol, and Harold Zakon, James Larimer, and Jane Lubischer for critical comments on this manuscript.

Correspondence should be addressed to Flora M. Love, Department of Zoology, University of Texas at Austin, Austin, TX 78712.

Copyright (C) 1998 Society for Neuroscience $\quad 0270-6474 / 98 / 189376-10 \$ 05.00 / 0$ process extension (Georgiou et al., 1994). In addition to their ability to sense and respond to changes in synaptic activity, TSCs also have the capacity to alter the structure of neuromuscular synapses. Processes growing from SCs implanted into the endplate zone of normal muscle, on contacting the endplates in the host muscle, alter the area of contact between the nerve terminal and muscle fiber (Trachtenberg and Thompson, 1997).

In view of the influence that TSCs can exert on motor axons and neuromuscular junctions, we undertook a study to determine how the number of these cells at neuromuscular junctions is regulated. We extend previous observations of SC addition to developing junctions (Love and Thompson, 1996; Hirata et al., 1997), relate TSC number to junctional size, describe SC mitosis at developing junctions, and report on the mitotic response of SCs in adult junctions to denervation, reinnervation, and partial denervation. These results show that there is a dynamic relationship between TSCs and motor neuron terminals during development, reinnervation, and sprouting.

\section{MATERIALS AND METHODS}

\section{Animals and surgery}

Developmental study. The soleus and extensor digitorum longus (edl) muscles were examined in Wistar rats at postnatal day $0(\mathrm{P} 0), \mathrm{P} 7$, and $\mathrm{P} 14$, in adults (2 months of age), and in older adults (15-17 months). Muscles were removed under deep ether anesthesia and immersed in oxygenated Ringer's solution (Liley, 1956).

Reinnervation. Animals were anesthetized by intraperitoneal injections of ketamine/xylazine. The soleus nerve was crushed $1 \mathrm{~mm}$ from entry into the soleus muscle in 5- to 6-week-old Wistar rats. The soleus muscles were examined 6-8 d later. Results were compared with those obtained in a separate set of animals in which the soleus was fully denervated for an equivalent period of time.

Partial denervation. The AO strain of Wistar rats in which innervation 
of the soleus is often derived from two separate nerves (Thompson and Jansen, 1977) was used. Partial denervation was accomplished by resecting one of the two nerves (the soleus nerve at the entry point into the muscle) in 5-week-old rats.

\section{Bromodeoxyuridine administration}

Bromodeoxyuridine (BrdU) (203806; Calbiochem, La Jolla, CA) dissolved in $0.9 \% \mathrm{NaCl}$ containing $0.007 \mathrm{~N} \mathrm{NaOH}$ was injected into the peritoneal cavity. A dose of $1 \mathrm{mg} / 10 \mathrm{gm}$ of body weight was administered to adults receiving multiple injections and to neonates. In adults receiving a single injection, $2 \mathrm{mg} / 10 \mathrm{gm}$ of body weight was given.

\section{Whole-mount immunolabeling}

After removal from the animal, the soleus and edl muscles were fixed in $4 \%$ paraformaldehyde. For ages P7 to adult, muscles were fixed for 10 min. For P0 and BrdU-treated animals, the time was extended to $20 \mathrm{~min}$ to allow for better fixation of S-100. Muscles were then rinsed for $30 \mathrm{~min}$ in three changes of $0.1 \mathrm{M} \mathrm{PBS}, \mathrm{pH} 7.4$, permeabilized in $-20^{\circ} \mathrm{C}$ methanol for 5-10 min (depending on the size of the muscle), washed again in PBS for $30 \mathrm{~min}$, and blocked in PBS containing 0.3\% Triton X-100, 0.2\% bovine serum albumin, and $0.1 \%$ sodium azide. In BrdU-treated animals, before blocking, muscles were denatured in $2 \mathrm{~N} \mathrm{HCl}$ in PBS containing $0.3 \%$ Triton X-100 for $30 \mathrm{~min}$ and subsequently washed for $30 \mathrm{~min}$ in three changes of PBS containing Triton X-100. Muscles were then incubated in primary antibodies overnight at room temperature. The following antibodies were used. SCs were labeled with rabbit anti-cow S-100 (Dako, Carpinteria, CA), diluted 1: 500; axons were labeled with mouse monoclonal antibodies to a neurofilament protein $(2 \mathrm{H} 3$ supernatant, Developmental Studies Hybridoma Bank), diluted 1:250; and nerve terminals were labeled with mouse monoclonal antibodies to synaptophysin (Sigma, S-5768; Sigma, St. Louis, MO), diluted 1:400. Cells that incorporated BrdU were labeled with a 1:5 dilution of mouse monoclonal antibodies to BrdU (G3G4 supernatant, Developmental Studies Hybridoma Bank). Proliferating cells were also labeled with mouse monoclonal antibodies to proliferating cell nuclear antigen (PCNA), diluted 1:1000 (Sigma, P-8835). In nerve injury studies, denervated endplates were identified by labeling with Cy5-conjugated bungarotoxin. After incubation in primary antibodies, muscles were rinsed in three changes of PBS for $30 \mathrm{~min}$ and incubated in secondary antibodies for $1 \mathrm{hr}$ at room temperature. The secondary for S-100 was rhodamine-conjugated goat $\mathrm{F}(\mathrm{ab})_{2}$ fragment anti-rabbit (Cappel, 55671), diluted 1:400. The secondary for the anti-synaptophysin, anti-neurofilament, BrdU, and PCNA antibodies was fluorescein-conjugated sheep $\mathrm{F}\left(\mathrm{ab}^{\prime}\right)_{2}$ fragment antimouse (Sigma, F-2266), diluted 1:100. After incubation with the secondary antibodies, the muscles were rinsed in PBS for $30 \mathrm{~min}$. Nuclei were then stained by placing the muscles in PBS containing 4',6-diamidino-2phenylindole (DAPI) $\left(10^{-4} \mathrm{mg} / \mathrm{ml}\right)$ for $7 \mathrm{~min}$.

After immunostaining, thin layers of the muscles were dissected and mounted in fluorescence mounting medium [formula of Johnson and de C Nogueira Araujo (1981), with 0.1 M ethanolamine substituted for PBS and PPD increased to $2 \mathrm{mg} / \mathrm{ml}$. The final $\mathrm{pH}$ of the solution was 9.5 (Swartz and Santi, 1996). The tissue was then examined using a Nikon microscope equipped for epifluorescence. Images were acquired using rhodamine, fluorescein, UV, and Cy5 filters and an integrating CCD camera connected to a Macintosh computer equipped with a frame grabber and running NIH Image software.

\section{Analysis}

The number and position of TSC nuclei were determined by the colocalization of labels for DAPI and S-100 over synaptophysin-labeled nerve terminals. Endplates in an en face orientation within the endplate zone were examined. A minimum of 30 endplates was examined from each animal. Although mitosis of SCs was observed on preterminal axons and in the intramuscular nerves, quantification was not possible because only short segments of axons were visible.

Measurements of the nerve terminal area were made in adult animals only at endplates at which there was no SC soma on the preterminal axon. In this manner, we eliminated the ambiguity of whether these cells should be counted in the TSC number because their processes could contribute to the coverage of the endplate. Areas were determined from digitized images by the use of NIH Image software running on a Macintosh computer. The gray scale image of the synaptophysin-labeled nerve terminal was converted to binary, and the threshold was adjusted for black/white to obtain a black image that by visual inspection encom- passed the nerve terminal. The area of the black pixels was then determined by the software.

Proliferation of SCs at the neuromuscular junction was examined by counting the number of TSCs that incorporated BrdU or were labeled with antibodies to PCNA (see above). It has been reported that in tissues fixed with aldehydes, PCNA labeling occurs throughout the cell cycle, whereas in tissues fixed with organic solvents, staining occurs only during DNA synthesis (Bravo and Macdonald-Bravo, 1987). Paraformaldehyde was necessary for fixation of the SC antigen S-100 but may have resulted in labeling of PCNA in the absence of replication. To verify that the PCNA staining occurred only in proliferating cells, tissues were fixed with methanol instead of paraformaldehyde, and the number of PCNAlabeled nuclei positioned directly over the nerve terminals was compared with the number of TSCs labeled with PCNA in paraformaldehyde-fixed muscles. Both soleus and edl muscles were removed from one P10 rat. In the soleus and edl muscle fixed with paraformaldehyde, $18 \%(12 / 67)$ and $23 \%$ (26/113), respectively, of the endplates had a cell that labeled with both PCNA and S-100. In the soleus and edl muscle fixed with methanol, $26 \%(23 / 89)$ and 22\% (30/136), respectively, of the endplates had PCNAlabeled cells overlying the nerve terminals. Thus, there were no more nuclei labeled in the paraformaldehyde-fixed muscles than in the methanol-fixed muscles.

\section{RESULTS}

\section{The number of SC somata at the endplate increases during postnatal development and differs between soleus and edl}

To examine developmental changes in the number of TSCs, rat soleus and edl muscles of various ages were triple-labeled with rabbit polyclonal antibodies identifying SCs (anti-S-100), a combination of two mouse monoclonal antibodies (anti-neurofilament and anti-synaptophysin) identifying axons and nerve terminals, and a nuclear label (DAPI). By the use of rhodamine- and fluorescein-conjugated secondary antibodies to rabbit and mouse antibodies and by examination with appropriate filters for DAPI, fluorescein, and rhodamine, individual synapses and the SCs covering them could be identified (Figs. 1,2). Comparison of the $\mathrm{SC}$ and nuclear labels allowed a determination of the number of SC somata present. At the time of birth, many endplates in soleus and edl (Fig. 1) had no SC somata. Counting only somata present at the endplate, an average of $0.44 \pm 0.12$ SCs (range, $0-2 ; 104$ endplates examined) were present at endplates in the newborn soleus, and $0.69 \pm 0.09$ (range 0-2; 96 endplates examined) were present in edl. Most $(99 / 102=97 \%)$ of those endplates lacking SC somata were covered by SC processes arising from a soma or somata located along the preterminal axon (Fig. 1).

Applying this same analysis to adult junctions (animals at 2 months of age) revealed that, on average, $2.7 \pm 0.3$ (range 1-7; 182 endplates examined) nuclei were present at junctions in soleus and $3.7 \pm 0.2$ (range 1-9; 248 endplates examined) were present in edl. Thus, in agreement with a previous report (Hirata et al., 1997), SCs are added to junctions during postnatal development. The most dramatic increase in TSC number occurs during the first 2 weeks of postnatal life (Fig. $2 G$ ). In soleus, the number of TSCs increased $384 \%$ from P0 to P14 but only $25 \%$ from P14 to 2 months of age. In edl, TSC number increased $272 \%$ from P0 to P14, but only $43 \%$ from P14 to 2 months of age. Examination of muscles at 15 months of age suggests that addition of TSCs continues even in the adult, but at a much slower pace than that seen during the first 2 months of age. Muscles at 15 months have gained one to two additional TSCs over those present at 2 months of age. 

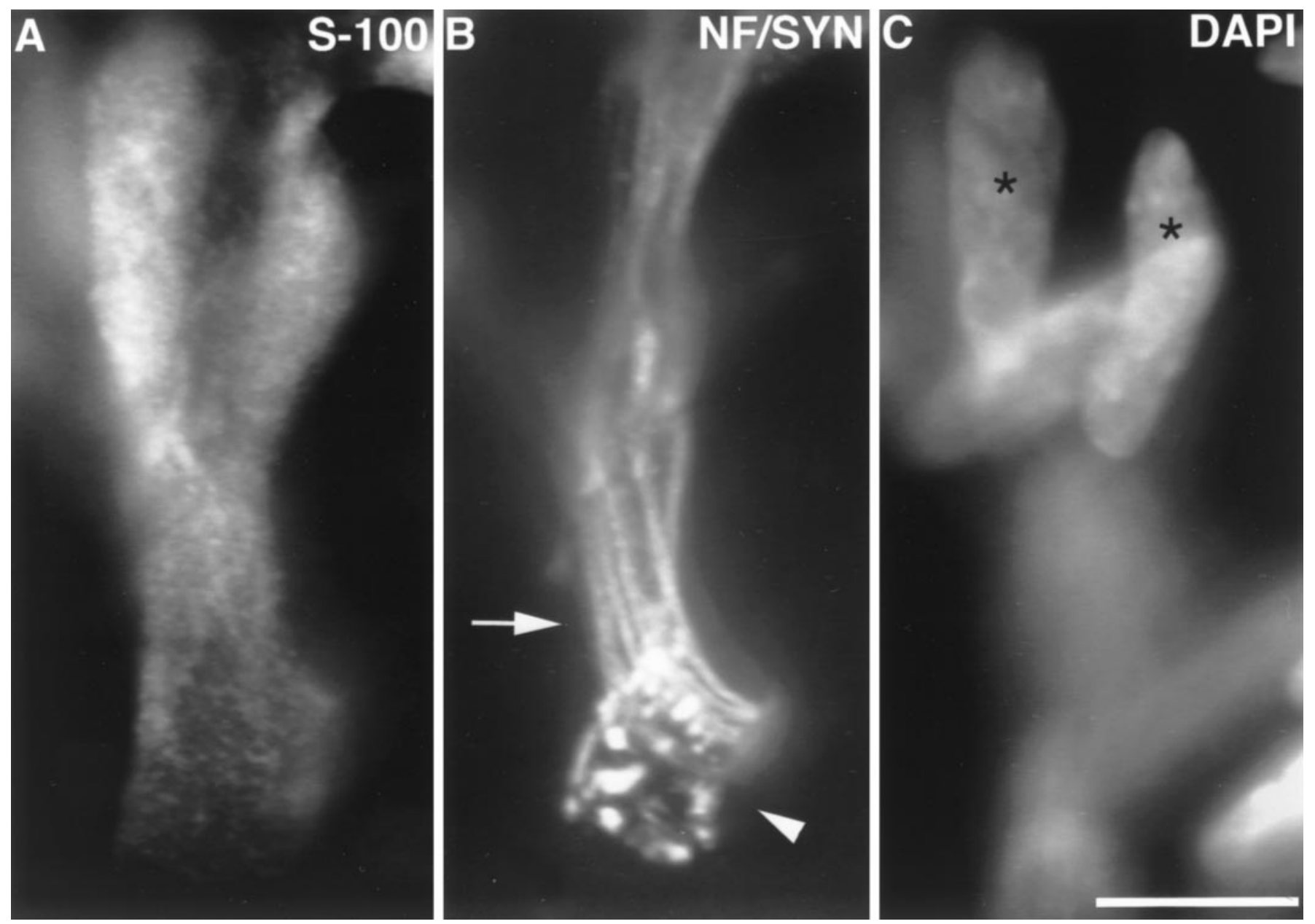

Figure 1. Image of an edl endplate at P0. At P0, approximately half of the endplates in soleus and edl have no SC somata. $A$, SCs labeled with anti-S-100 antibodies; $B$, preterminal axons (arrow) labeled with anti-neurofilament antibodies $(N F)$, and nerve terminal (arrowhead) labeled with antisynaptophysin $(S Y N)$ antibodies; $C$, nuclei labeled with $D A P I$. Several preterminal axons enter the endplate $(B)$, showing the polyneuronal innervation present at this age. Despite the presence of SC processes covering the terminal, no SC somata are present at the junction. Rather, two SC somata (asterisks) are located on the preterminal axons. DAPI and S-100 images were taken in the same focal plane. Other nuclei, oriented horizontally, are likely to be muscle nuclei. Scale bar, $10 \mu \mathrm{m}$.

\section{TSC number is correlated with terminal area, but most of the gain in TSC number precedes the gain in endplate area}

One possible explanation of the increase in TSC number with development is the growth in the size of endplates with age as muscle fibers expand in diameter (cf. Balice-Gordon et al., 1990). To examine the correlation between endplate area and TSC number, endplate area (measured as the terminal arborization labeled with anti-synaptophysin antibody) and TSC numbers were determined for junctions in muscles from 2-month-old adults. There was a tendency for larger endplates to have more SCs, both in soleus and edl (Fig. 3A). The correlation coefficient $(r)$ for TSC number and endplate size was 0.37 for soleus and 0.68 for edl. Both numbers are significantly different $(p<0.01)$ from what would be expected for a random relationship between area and TSC number. However, the proportion of the variation in TSC number that can be accounted for by endplate area (the square of the correlation coefficient) is only $14 \%$ for soleus and $46 \%$ for edl.

Additional observations of the relationship between endplate area and TSC number were made in animals during postnatal development, a time in which there is a dramatic increase in the endplate area (cf. Slater, 1982; Balice-Gordon and Lichtman,
1990; Waerhaug, 1992). As reported above, most of the addition of TSCs occurs in the first 2 postnatal weeks. Likewise, endplate area increases by approximately fourfold from birth to adulthood; however, most of this increase occurs after P14 (Fig. 3B). Thus, the increase in endplate area lags behind the increase in TSC number. In soleus, for example, there is a fourfold increase in TSC number during the period from birth to P14 when the endplate area increases less than twofold. Between P14 and P60, the endplate area increases by more than twofold, but the TSC number increases only $25 \%$. Therefore, expanding endplate area may not be the major change driving the increase in TSC number.

\section{Migration and mitosis account for the addition of SCs to developing junctions}

As stated above, $\sim 30-55 \%$ of the endplates in edl and soleus have no TSCs at the time of birth. Thus, the initial increase cannot occur by mitosis of SCs at the endplate. Two other possibilities exist. (1) SCs may migrate into the endplate along preterminal axons, or (2) TSCs may differentiate from S-100negative precursor cells located at the endplate. This latter possibility, however, is not likely given that many newborn junctions lack any nuclei overlying the nerve terminal (compare Fig. 1). It 

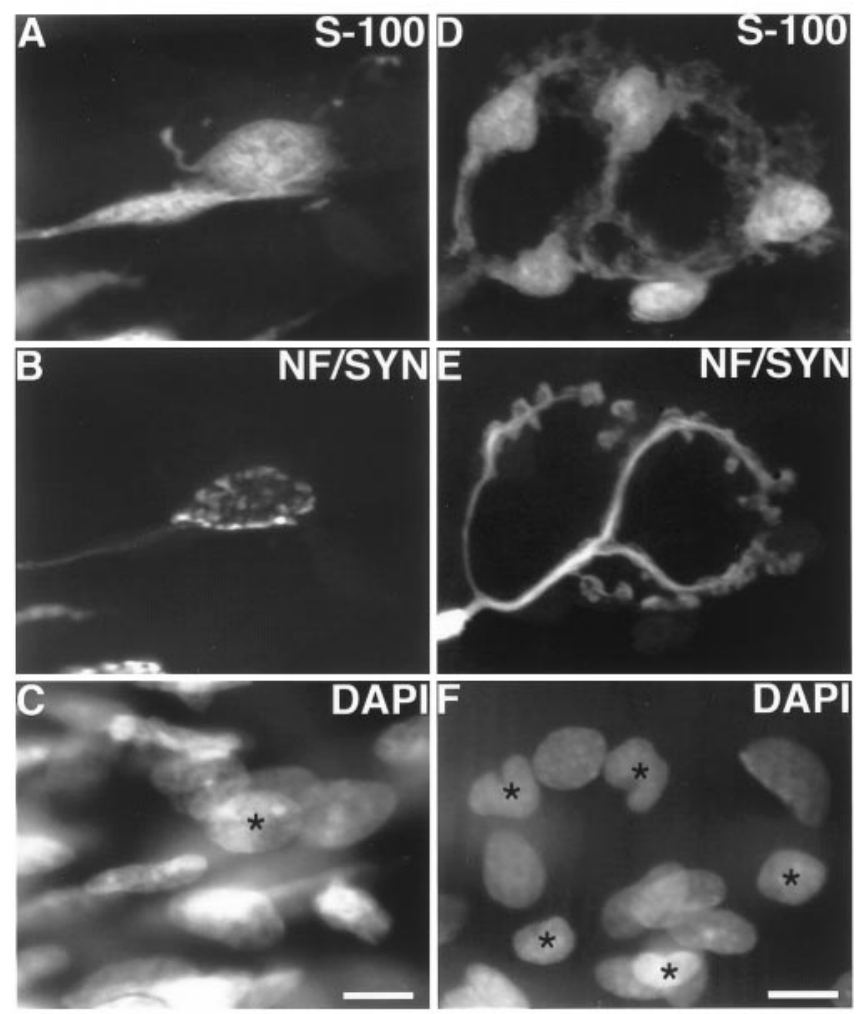

G

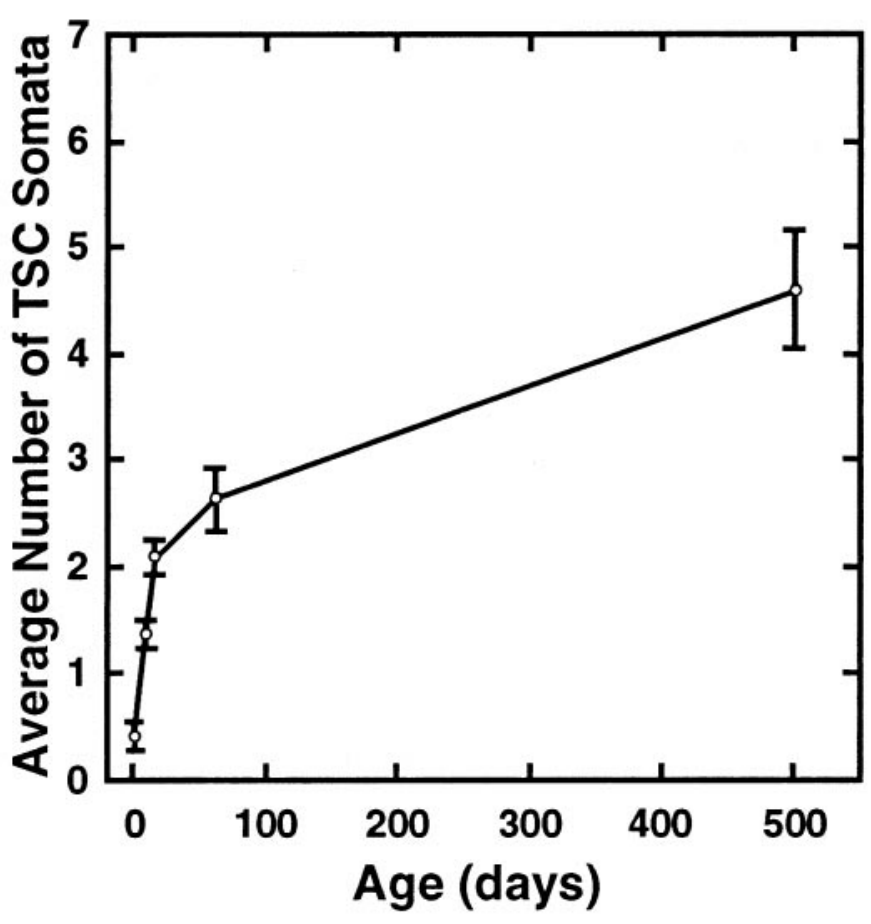

Figure 2. The number of TSC somata increases during postnatal development and adulthood. Image of one edl endplate at P7 $(A-C)$ and one at 2 months $(D-F) . A, D$, TSCs labeled with anti-S-100 antibodies; $B, E$, preterminal axon and nerve terminal, labeled with anti-neurofilament and anti-synaptophysin antibodies; $C, F$, nuclei labeled with DAPI. Asterisks mark the nuclei of cells that colabel with S-100. This P7 endplate has one TSC soma compared with five TSC somata at the adult endplate. Scale bars, $10 \mu \mathrm{m}$. $G$, Line graph of TSC number at P0, P7, P14, 2 months, and appears, therefore, that some of the SCs located along the preterminal axons that extend processes to cover the endplate migrate into the endplate subsequent to the time of birth.

Such migration could account for all of the TSCs present at junctions. Alternatively, division of TSCs at the junction could contribute to the rise in number. Therefore, an attempt was made to determine whether TSCs underwent mitoses at developing junctions. Preliminary experiments were performed in which the mitotic label BrdU was injected one to three times, 90 min apart, in two P10 rats, and the muscles were removed $9 \mathrm{hr}$ after the first injection. BrdU-positive nuclei that were also S-100-positive were present at $10 \%$ (40 of 398) of the endplates in soleus and 14\% (43 of 305) of those in edl. Most of these endplates had only a single labeled nucleus. However, 12 of the 40 endplates in soleus and 16 of the 43 in edl had two labeled nuclei in close apposition, suggesting that these were daughter cells that had completed mitosis. The small number of nuclear pairs is not surprising considering that only $9 \mathrm{hr}$ had elapsed since the first BrdU injection and because estimates of the length of $G 2+M$ (the time between the end of synthesis and the end of mitosis) for SCs in the peripheral nerves of mice is $\sim 4-8 \mathrm{hr}$ (Asbury, 1967; Usson and Saxod, 1988). Thus, only those cells that were in the late phase of DNA synthesis at the time of the BrdU injection would have completed mitosis. These observations show that mitosis contributes to the rise in SC number at developing endplates. Although the presence of pairs of labeled TSC nuclei at single endplates $9 \mathrm{hr}$ after injection of BrdU suggests that TSCs may undergo mitosis at the endplate, it is difficult to exclude the possibility that the labeled cells divided somewhere along the nerves and then migrated into the endplate. Similarly, it is difficult to argue that a single labeled nucleus at the endplate indicates DNA synthesis at this site, because only one of the daughters of cells completing mitosis may have migrated into the endplate.

To determine where SCs that come to cover the nerve terminal divide, a more quantitative analysis was made after a single injection of BrdU into three P8 animals, 30 min before they were killed. Our reasoning was that this would be an insufficient time (given the interval for $G 2+M$ ) for cells to complete mitosis and migrate into the junctions. In three animals, 4,5 , and $6 \%$ (average, $5 \%$; 488 endplates examined) of the endplates in soleus and 6, 9, and $10 \%$ (average, $8 \%$; 198 endplates examined) in edl had a TSC labeled by BrdU (Fig. 4). No BrdU-labeled cells were encountered after a similar 30 min labeling in muscles at 5 weeks $(n=3)$.

The numbers of TSCs labeled by a 30 min exposure to BrdU appear quite reasonable given our earlier determination of the numbers of SCs added to junctions. From simply counting SCs at junctions, we determined that $\sim 0.14 \mathrm{SCs}$ are added each day to each junction in soleus and 0.11 in edl. Assuming that TSCs, like their counterparts in the nerve, have an $8 \mathrm{hr}$ DNA synthesis period and a $24 \mathrm{hr}$ cell cycle (Asbury, 1967), one-third (i.e., 8/24) of these cells should be undergoing DNA synthesis at any moment in time and would be expected to be labeled by injections of BrdU over a 30 min period. This leads to the prediction that $4.7 \%$ $(0.14 / 3)$ of the TSCs should incorporate BrdU in soleus and $3.7 \%$

15-17 months in soleus. The number of TSCs was determined by colocalizing DAPI and S-100 labels over synaptophysin-labeled regions. Three rats from each age group were examined, and a minimum of 30 endplates was examined in each muscle. An ANOVA for the change in TSC number with age was significant for both soleus and edl $(p<0.005)$. 

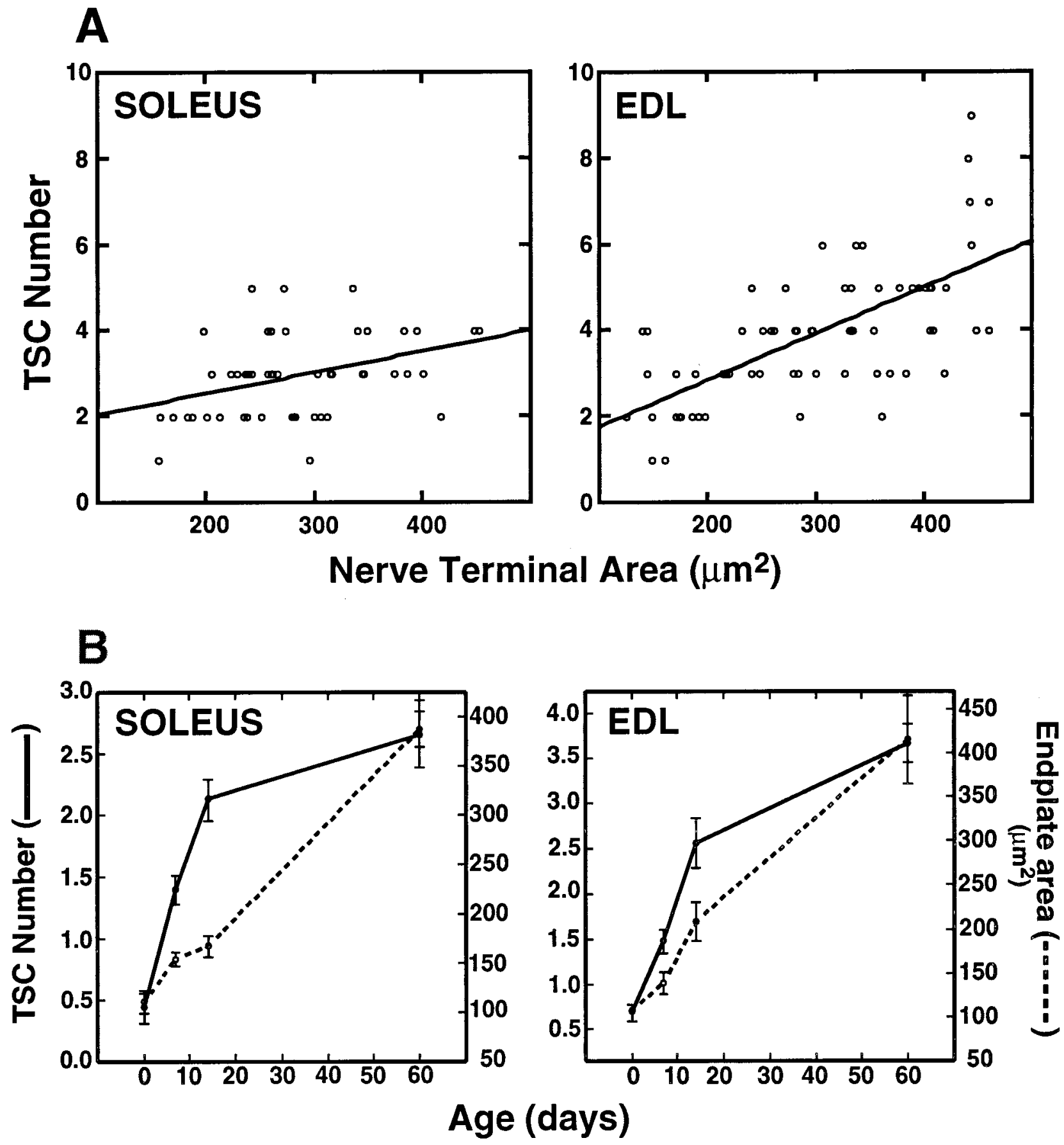

Figure 3. TSC number is correlated with terminal area, but most of the postnatal gain in TSC number precedes the gain in endplate area. $A$, Correlation of nerve terminal area and TSC number in 2-month-old soleus and edl muscles. The correlation coefficient $(r)$ was 0.37 for soleus and 0.68 for edl $R^{2}=0.14$ for soleus and 0.46 for edl. Both are significant with $p<0.01$. Nerve terminal area was determined by measuring the area labeled by anti-synaptophysin antibodies. TSC number was determined by colocalization of S-100 and DAPI labeling over the nerve terminal region. $B$, Change in TSC number (solid line) and endplate area (hatched line) with age in soleus and edl. Data for the change in TSC number with age in soleus are replotted from Figure $2 G$. Endplate area was determined by measuring the area labeled by binding of rhodamine-conjugated $\alpha$-bungarotoxin in one set of muscles. The number of TSCs was determined in a second set of muscles by colocalization of DAPI and S-100 labels over synaptophysin-labeled regions. In each set of muscles, three rats from each age group were examined, and a minimum of 30 endplates was examined in each muscle.

in edl. Thus, the observed labeling is reasonably consistent with that anticipated if mitosis at the junction accounts for most of the addition of SCs to junctions.

To verify the results obtained using BrdU, muscles were also labeled with antibodies to PCNA, an auxiliary protein of DNA polymerase whose expression in the nucleus increases during
DNA synthesis (Bravo and Macdonald-Bravo, 1987). Labeling of TSCs was seen at all of the early postnatal ages examined (P0, P7, $\mathrm{P} 8$, and P10). A quantitative analysis was performed at P10 in one rat. In soleus, $17 \%$ (12 of 69) of the endplates had a TSC labeled with PCNA. In edl, $22 \%$ (30 of 136) of the endplates had a PCNA-labeled TSC. In cells fixed with paraformaldehyde, stain- 


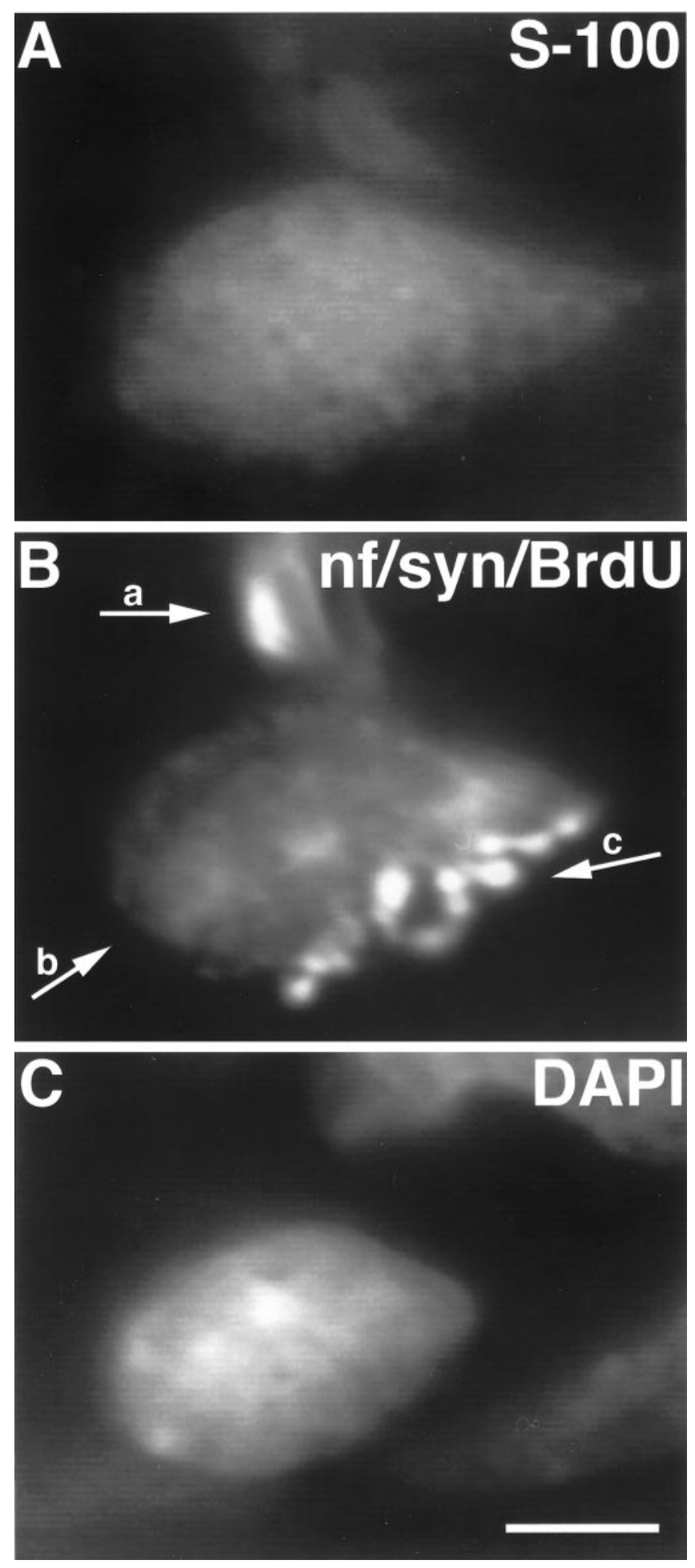

Figure 4. Image of a TSC at a P8 soleus endplate labeled with BrdU. SCs divide at the neuromuscular junction during early postnatal development. $A$, TSC, labeled with antibodies to S-100; $B$, preterminal axon $(a)$ labeled with anti-neurofilament antibodies, SC nucleus $(b)$ labeled with antibodies to BrdU, nerve terminal (c) labeled with anti-synaptophysin antibodies; $C$, SC nucleus labeled with DAPI. The BrdU antibodies and the antibodies used to detect axons and nerve terminals were all mouse monoclonals. Thus, mitotic nuclei, axons, and terminals were labeled using the same FITC-conjugated secondary antibody. Although the endplate in $B$ is labeled with three primary antibodies, the BrdU-labeled nucleus (arrow $b$ ) is easily distinguished from the nerve terminal and the preterminal axon. Scale bar, $10 \mu \mathrm{m}$.
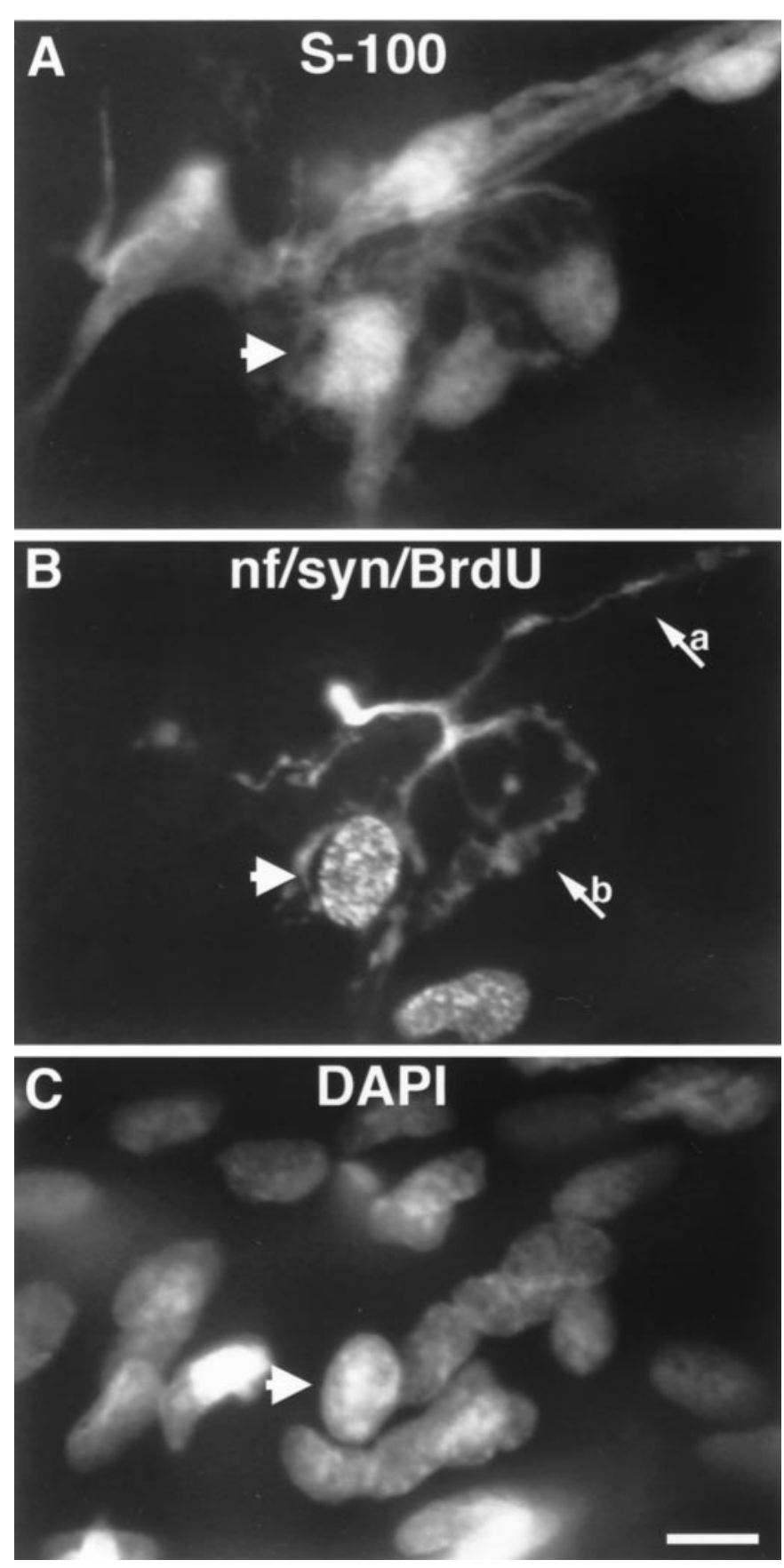

Figure 5. Image of a TSC labeled with BrdU at a reinnervated endplate $7 \mathrm{~d}$ after nerve crush. Proliferation of TSCs occurs during reinnervation after nerve injury. $A$, SCs labeled with anti-S-100 antibodies; $B$, mitotic cells are labeled with antibodies to $\mathrm{BrdU}$, and the preterminal axon $(a)$ and nerve terminal $(b)$ are labeled with anti-neurofilament and antisynaptophysin antibodies; $C$, nuclei labeled with DAPI. A TSC that labels with BrdU is indicated by the arrowhead present in each panel. An additional BrdU-labeled nucleus can be seen adjacent to the endplate that does not belong to a TSC (compare $A, B$ ). Scale bar, $10 \mu \mathrm{m}$.

ing can occur throughout the cell cycle and is present in the cytoplasm for $\sim 24 \mathrm{hr}$ after mitosis (Bravo and Macdonald-Bravo, 1987). Thus, it is not surprising that more cells were labeled by PCNA than by a single injection of BrdU. Furthermore, some of the PCNA-labeled cells may represent those that divided on the nerve and subsequently migrated to the endplate. No PCNA labeling of TSCs was observed in muscles at 3 months of age $(n=3)$. 


\begin{tabular}{|c|c|c|c|c|c|}
\hline \multicolumn{3}{|c|}{$\begin{array}{l}\text { Table 1. Mitotic TSCs in six rat soleus m } \\
\text { min before rats were killed } \\
\text { Innervated endplates without } \\
\text { bridges } \\
\end{array}$} & \multicolumn{2}{|c|}{$\begin{array}{l}\text { Innervated endplates with } \\
\text { bridges }\end{array}$} & \multirow[b]{2}{*}{$\begin{array}{l}\text { Ratio of innervated to } \\
\text { denervated endplates }\end{array}$} \\
\hline & Total & $\begin{array}{l}\text { Number and percent } \\
(\%) \text { with BrdU label }\end{array}$ & Total & $\begin{array}{l}\text { Number and percent } \\
(\%) \text { with BrdU label }\end{array}$ & \\
\hline 1 & 29 & $2(7)$ & 13 & $6(46)$ & $42: 202(0.21)$ \\
\hline 2 & 146 & $13(9)$ & 27 & $8(30)$ & $173: 159(1.09)$ \\
\hline 3 & 39 & $1(3)$ & 9 & $1(11)$ & $48: 243(0.20)$ \\
\hline 4 & 22 & $6(27)$ & 12 & $3(25)$ & $34: 294(0.11)$ \\
\hline 5 & 119 & $9(8)$ & 19 & $4(21)$ & $138: 144(0.96)$ \\
\hline 6 & 44 & $2(5)$ & 15 & $7(47)$ & $59: 310(0.19)$ \\
\hline Total & 399 & $33(8)$ & 95 & $29(31)$ & \\
\hline
\end{tabular}

No BrdU-labeled nuclei were seen at denervated endplates, even those associated with bridges.

\section{In transiently denervated muscles, TSCs divide, not in response to denervation but during reinnervation}

TSCs appear to play important roles in the repair of nerve damage and the reinnervation of muscle fibers. Therefore, we wished to know whether proliferation of these cells accompanied muscle denervation and reinnervation. For this purpose, the soleus muscle was denervated by nerve crush or resection in 5- to 6-week-old rats. At $6 \mathrm{~d}$ after nerve crush, axon terminals had returned to all the junctions. In contrast, no reinnervation was observed in muscles in which a piece of the nerve was resected, even $8 \mathrm{~d}$ after resection. At 6, 7, and $8 \mathrm{~d}$ after crush or resection, animals received a single injection of BrdU 30 min before they were killed. In muscles that were denervated, no BrdU-positive TSCs were seen in a total of 386 endplates examined (three animals). Thus, denervation per se did not promote SC mitosis at junctions. In contrast, all three animals that were being reinnervated after nerve crush had BrdU-positive TSCs (Fig. 5). On average, $16 \pm 5 \%$ (365 endplates examined) had at least one BrdU-labeled TSC. Because only $30 \mathrm{~min}$ had elapsed after the BrdU injection, it is unlikely that these cells had migrated into the junctions; rather, it appears that they became mitotic on the junction itself.

Antibodies to PCNA were also used to identify mitotic cells in denervated and reinnervated muscles. In two animals, $6 \mathrm{~d}$ after soleus nerve crush, $28 \%$ (22 of 77 ) and 40\% (29 of 72) of reinnervated endplates had at least one PCNA-positive TSC. In contrast, none of the 165 endplates examined in two muscles denervated by nerve resection for $6 \mathrm{~d}$ had PCNA-labeled TSCs. As in the neonates, the percentages of cells labeled with PCNA are higher than with BrdU and may reflect the presence of detectable levels of PCNA beyond the period of DNA synthesis.

\section{After partial denervation, SCs proliferate at innervated endplates, particularly those connected to denervated endplates by SC bridges}

TSCs play a major role in the nerve sprouting that follows damage to a portion of the axon supply to the muscle, i.e., in response to partial denervation. A great deal of the growth of nerve sprouts occurs along processes extended from TSCs at denervated endplates that come into contact with innervated endplates, i.e., along SC bridges (Son and Thompson, 1995b). To examine how partial denervation affects TSC mitotic activity, one of two nerves supplying the soleus was resected so that only a few of the motor axons to the muscle remained. In a preliminary set of experiments using two 5-week-old rats, BrdU was injected at multiple times on each of the $3 \mathrm{~d}$ after partial denervation. When the soleus muscles were examined on the fourth day, BrdUlabeled SCs were found at 18 of 67 innervated endplates and 2 of 128 denervated endplates. These two examples at denervated endplates, however, represented a special case. They were connected to innervated endplates by a SC bridge, resulting in their reinnervation by terminal sprouts. In addition, 12 of the 18 innervated endplates with mitotic TSCs were connected to adjacent denervated synaptic sites by a SC bridge. In total, there were 22 pairs of endplates connected by bridges. Of these, 12 had a BrdU-labeled SC on the innervated endplate, two had BrdUlabeled SCs located on the bridge linking the two endplates, and two had a mitotic SC on the denervated endplate. These results suggest that TSC proliferation is a consequence of partial denervation and in most cases is associated with bridge formation. Given the long-term presence of BrdU and the possibility of SC migration, these experiments did not unambiguously identify the site where SCs became mitotic.

To examine where SCs divide in partially denervated muscles, partial denervations were performed on soleus muscles in six 5-week-old animals. A single BrdU injection was given 3 d later, $30 \mathrm{~min}$ before the animals were killed. BrdU-labeled TSCs were found at 62 of 494 innervated endplates but were absent from all denervated endplates examined (1352). Because SC mitosis was not found in control muscles at 5 weeks of age (see above), it appears that the denervated fibers (or, alternatively, denervated SCs or degenerating axons) in these partially denervated muscles promote mitosis of those TSCs that remain in contact with the nerve. Closer examination of the innervated endplates showing SC mitosis revealed that many of these endplates were linked by SC processes to nearby denervated endplates. In total, regardless of mitotic state, 95 innervated endplates in these muscles had been bridged to an adjacent denervated endplate by SC processes; nerve sprouts were present on all of these bridges. Of these 95 pairs of endplates, 29 had BrdU-labeled TSCs at the innervated endplate (Table 1, Fig. 6). Thus, 47\% (29/62) of the labeled TSCs were found at $19 \%(95 / 494)$ of the innervated endplates that had received this SC contact or bridge. Thus, a disproportionate share of the mitotic TSC population is present at these special endplates. In contrast, there were no BrdU-labeled TSCs on the bridge linking the endplates or on the denervated endplates 

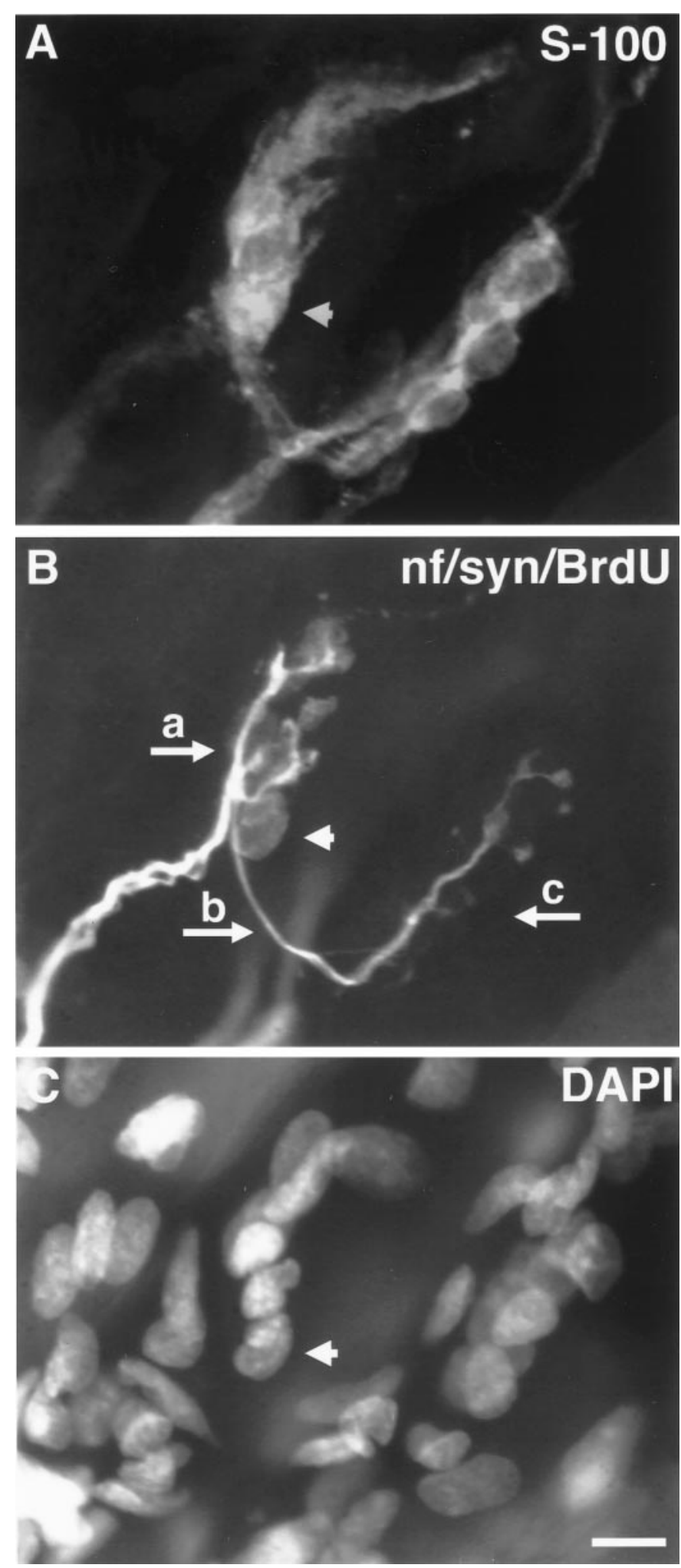

Figure 6. Image of a case of terminal sprouting in a soleus muscle $3 \mathrm{~d}$ after partial denervation. After partial denervation, SCs proliferate at innervated endplates, especially those connected to denervated endplates by SC bridges. $A$, SCs labeled with anti-S-100 antibodies; $B$, mitotic cells labeled with antibodies to BrdU; preterminal axon and nerve terminal are labeled with anti-neurofilament and anti-synaptophysin antibodies. $C$, Nuclei labeled with DAPI. The muscle was also labeled with Cy5conjugated bungarotoxin (data not shown), allowing identification of denervated endplates. Note that the innervated endplate $(a)$ is connected connected to an innervated endplate by a SC bridge. This result suggests that SCs growing from denervated synaptic sites can stimulate, by contact, mitosis of the SCs at innervated endplates either directly or indirectly by the induction of nerve terminal sprouting. Of the innervated endplates that had mitotic TSCs but were not connected to denervated endplates by SC bridges, many, but not all, were sprouting. Another possibility is that the mitotic activity in these SCs (and the other SCs not contacted by a bridge) is attributable to proximity to a denervated muscle fiber or a denervated synaptic site. If so, this explanation would require the frequency of bridges to be a function of proximity of denervated and innervated synaptic sites. Because of the way the muscles were prepared for examination in this study (as thin layers of fibers cut from the muscle that were then flattened), the spatial interrelationships in the original muscle could not be determined.

\section{DISCUSSION}

TSCs appear to be important in the reinnervation of neuromuscular junctions after nerve damage (Son and Thompson, 1995a,b). Moreover, reactive SCs, i.e., SCs responding to the loss of nerve contact, can produce major alterations in the structure of the adult neuromuscular synapse (Trachtenberg and Thompson, 1997). Knowledge of when these cells appear at junctions during development and how they respond to nerve injury is therefore important for understanding the formation and maintenance of this synapse. We have investigated changes in the SC coverings of the nerve terminal at neuromuscular synapses during development and regeneration. We find that these cells undergo mitosis during early development, during muscle reinnervation, and during nerve sprouting after partial denervation. Muscle denervation appears to provide a stimulus for division, but this stimulus is only effective when the SCs are in contact with an axon. This implies that the nerve supplies some signal required for TSC division. Furthermore, this stimulus for mitosis is greatly amplified by the contact of the nerve terminal with reactive SC processes from denervated endplates.

\section{Addition of TSCs during development}

TSCs extend processes after complete and partial denervation that appear to guide the growth of axons during reinnervation and sprouting (Son and Thompson, 1995a,b). Whether SCs play a similar guidance role during development has been a subject of some controversy (Keynes, 1987). Numerous investigators have used electron microscopy to examine the morphology of developing neuromuscular junctions. In mammals, the processes of SCs are present at junctions as they form in the embryo (Kelly and Zacks, 1969). However, SCs appear to be absent from some nascent junctions in birds (Jacob and Lentz, 1979) and Xenopus (Kullberg et al., 1977). Studies that have examined the role of SCs in axon guidance during embryonic development have also produced conflicting results (Harrison, 1924; Yntema, 1943; Noakes and Bennett, 1987; Dahm and Landmesser, 1988). The more recent use of mouse mutants (Grim et al., 1992) and mouse knock-outs (Reithmacher et al., 1997) that delete SCs strongly

$\leftarrow$

by a terminal sprout $(b)$ and the associated SC bridge to an endplate that was denervated $(c)$, resulting in its reinnervation. A TSC located at the innervated endplate is labeled with BrdU (arrowheads). Scale bar, $10 \mu \mathrm{m}$. 
suggests that SCs are not needed for the initial navigation of axons into the periphery or perhaps even for initial formation of neuromuscular junctions. However, SCs do play an important role in the maintenance of axons in early postnatal development in that motor neurons die in mice lacking SCs (Reithmacher et al., 1997).

In our study, SCs or SC processes, identified by immunohistochemistry for the SC marker S-100, were found to be present at $99 \%(197 / 200)$ of the neuromuscular junctions in two hindlimb muscles of the newborn rat. It is not clear why $1 \%$ were devoid of TSC processes. One possible explanation may be variations in the penetrance of the antibodies to S-100. This possibility seems unlikely, however, considering that the preterminal axons and TSCs at nearby endplates within the same focal plane were clearly labeled. Other possibilities are that SC processes have not yet reached the terminal or that a small proportion of SCs undergo apoptosis (cf. Trachtenberg and Thompson, 1996).

The results presented here show that junctions gain SCs as they mature. Initially this gain must be accounted for by migration. Such migration is not surprising given that it has been shown to be a primary mechanism accounting for population of developing nerves with SCs (Peters and Muir, 1959; Speidel, 1964). Although migration must be invoked to get the first cells to the junction, mitotic labeling shows that division of SCs also accounts for the addition. Comparison of the rate of SC addition to junctions and the extent of mitotic labeling of TSCs suggests that a considerable amount of this postnatal mitosis occurs at the junctions. During the first 2 postnatal weeks, TSC number expands three- to fourfold. The addition of SCs continues at a slower rate in the adult animal, and this addition appears to be correlated, in part, with the growth in the size of the endplate. This shows that whatever mechanisms exist to enlarge the nerve terminal as the muscle fiber grows may (directly or indirectly) increase the number of SCs to cover the expanded nerve terminal. However, it is likely that additional mechanisms determine TSC number, particularly in early postnatal development during which the largest gain in TSC number precedes most of the growth in endplate area.

\section{Addition of SCs during repair of muscle innervation}

We also investigated the addition of SCs to neuromuscular junctions during denervation, reinnervation, and nerve sprouting. Denervation alone failed to promote mitosis of TSCs. Our observations are entirely consistent with those of Connor et al. (1987), who reported that denervation produced mitosis of satellite cells in the junctional region of frog muscles but did not induce mitosis of TSCs. However, our results contrast with some reports for mitosis of nonmyelinating SCs in the nerve. SC mitosis has been reported in nonmyelinated nerves after injury, although more mitoses are reported in nerves containing both myelinated and nonmyelinated fibers (Romine et al., 1976; Salzer and Bunge, 1980). A recent study has shown that nonmyelinating $\mathrm{SCs}$ in the rat sciatic nerve, which contains both myelinated and nonmyelinated axons, proliferate to an extent similar to myelinating SCs during nerve degeneration (Clemence et al., 1988). The reason that TSCs, which are nonmyelinating, would differ from nonmyelinating SCs in the nerve is unclear.

Although denervation alone does not result in TSC mitosis, it does appear to provide some stimulus for mitosis considering that TSC mitosis was not seen in normally innervated adult muscle. Thus, axons regenerating into denervated muscles stimulate mitosis, and in partially denervated muscles, some SCs at the remaining innervated endplates undergo mitosis. In both of these cases, nerves are present, implying that a nerve-derived factor, in addition to denervated muscle fibers and/or degenerating nerves, is required. Clear evidence for a nerve-derived mitogenic signal has been obtained for SC-axon interactions in vitro (Wood and Bunge, 1975; Salzer et al., 1980a,b) and in vivo (Pellegrino and Spencer, 1984). The factor neuregulin has been shown to be a candidate for this axonal signal (Morrissey et al., 1995). Other possible neuron-derived SC mitogens include PDGF, FGF, and calcitonin gene-related peptide (CGRP) (Eccleston, 1992; Hokfelt et al., 1994; Cheng et al., 1995). Our experiments suggest that reactive TSCs also provide some kind of stimulus promoting TSC mitosis. Mitoses at innervated synaptic sites in partially denervated muscles were more frequent at sites that had been contacted by a process extended by a reactive TSC, i.e., an SC present at a nearby denervated synaptic site. This suggests that a reactive TSC stimulates division either by direct interaction with the SC at the innervated synaptic site or indirectly by its promotion of nerve growth by the nerve terminal. One possible candidate for an SC-derived mitogenic signal may be TGF- $\beta$. Schwann cells both synthesize and possess receptors for TGF- $\beta$ s (Scherer and Salzer, 1996). Another possible SC-derived mitogenic signal is neuregulin, a factor produced and secreted by SCs both in vitro (Raabe et al., 1996) and in vivo (Carroll et al., 1997).

In cases of nerve injury, the promotion of TSC mitosis appears to make functional sense. Division during reinnervation could function to increase the amount of SC membrane available to cover the reforming nerve terminal, perhaps compensating for SCs that migrate from the junctions during the time of denervation or for the SC processes that extend away from the junction. In partially denervated muscles, stimulation of SC division may provide additional SC membrane necessary for the wrapping of nerve sprouts. However, SC process extension does not require cell division, because TSCs extend long and numerous processes after denervation (Reynolds and Woolf, 1992).

\section{TSCs and synaptic stability}

In several cases, TSC division appears to be correlated with changes in the structure of the neuromuscular synapse. During the first 2 weeks of postnatal development in the rat, synapse elimination reduces the number of motor neurons innervating each fiber from several to one. During this period, the SC number increases three- to fourfold at junctions. As new SCs are generated and take up residence at the endplate, $\mathrm{SC}$-nerve terminal contact must also be rearranged. An EM study has shown that during this period, SC processes intervene between and separate axon terminals (Korneliussen and Jansen, 1976). Pomeroy and Purves (1988), observing vitally the movements of synaptic glial cells in mouse parasympathetic ganglia, suggested that these glial movements were involved in synaptic rearrangements. The neuregulin glial growth factor, suggested as an axon-derived mitogenic signal for SCs (Morrissey et al., 1995), if applied to developing neuromuscular junctions induces SC proliferation and decreases synaptic contacts at neuromuscular junctions (Trachtenberg and Thompson, 1997). Reactive TSC processes contacting innervated endplates in adult muscle were shown in the present study to cause TSC mitosis; a previous study has shown that these reactive SCs alter the apposition of nerve terminal and muscle fibers (Trachtenberg and Thompson, 1997). Whether the changes in synaptic glia are a consequence or a cause of synaptic change awaits further experimentation. 


\section{REFERENCES}

Asbury AK (1967) Schwann cell proliferation in developing mouse sciatic nerve. A radioautographic study. J Cell Biol 34:735-743.

Balice-Gordon RJ, Lichtman JW (1990) In vivo visualization of the growth of presynaptic and postsynaptic elements of neuromuscular junctions in the mouse. J Neurosci 10:894-908.

Balice-Gordon RJ, Breedlove SM, Bernstein S, Lichtman JW (1990) Neuromuscular junctions shrink and expand as muscle fiber size is manipulated: in vivo observations in the androgen-sensitive bulbocavernosus muscle of mice. J Neurosci 10:2660-2671.

Bravo R, Macdonald-Bravo H (1987) Existence of two populations of cyclin/proliferation cell nuclear antigen during the cell cycle: association with DNA replication sites. J Cell Biol 105:1549-1554.

Carroll SL, Miller ML, Frohnert PW, Kim SS, Corbett JA (1997) Expression of neuregulins and their putative receptors, erbB2 and erbB3, is induced during Wallerian degeneration. J Neurosci 17:1642-1659.

Cheng L, Khan M, Mudge AW (1995) Calcitonin gene-related peptide promotes Schwann cell proliferation. J Cell Biol 129:789-796.

Clemence A, Mirsky R, Jessen KR (1988) Non-myelin-forming Schwann cells proliferate rapidly during Wallerian degeneration in the rat sciatic nerve. J Neurocytol 18:185-192.

Connor EA, McMahan UJ, Marshall RM (1987) Cell accumulation in the junctional region of denervated muscle. J Cell Biol 104:109-120.

Dahm LM, Landmesser LT (1988) The regulation of intramuscular nerve branching during normal development and following activity blockade. Dev Biol 130:621-644.

Eccleston PA (1992) Regulation of Schwann cell proliferation: mechanisms involved in peripheral development. Exp Cell Res 99:1-9.

Georgiou J, Robitaille R, Trimble WS, Charlton MP (1994) Synaptic regulation of glial protein expression in vivo. Neuron 12:443-455.

Grim M, Halata Z, Franz T (1992) Schwann cells are not required for guidance of motor nerves in the hindlimb in Splotch mutant mouse embryos. Anat Embryol 186:311-318.

Harrison R (1924) Neuroblast versus sheath cells in the development of peripheral nerves. J Comp Neurol 37:123-205.

Hirata K, Zhou C, Nakamura K, Kawabuchi M (1997) Postnatal development of Schwann cells at neuromuscular junctions, with special reference to synapse elimination. J Neurocytol 26:799-809.

Hokfelt T, Zhang X, Wiesenfeld-Hallin Z (1994) Messenger plasticity in primary sensory neurons following axotomy and its functional implications. Trends Neurosci 17:22-30.

Jacob M, Lentz TL (1979) Localization of acetylcholine receptors by means of horseradish peroxidase-alpha-bungarotoxin during formation and development of the neuromuscular junction in the chick embryo. J Cell Biol 82:195-211.

Jahromi BS, Robitaille R, Charlton MP (1992) Transmitter release increases intracellular calcium in perisynaptic Schwann cells in situ. Neuron 8:1069-1077.

Johnson GD, de C Nogueira Araujo GM (1981) A simple method of reducing the fading of immunofluorescence during microscopy. J Immunol Methods 43:349-350.

Kelly AM, Zacks SI (1969) The fine structure of motor endplate morphogenesis. J Cell Biol 42:154-169.

Keynes RJ (1987) Schwann cells during neural development and regeneration: leaders or followers? Trends Neurosci 10:137-139.

Korneliussen H, Jansen JKS (1976) Morphological aspects of the elimination of polyneuronal innervation of skeletal muscle fibres in newborn rats. J Neurocytol 5:591-604.

Kullberg RW, Lentz TL, Cohen MW (1977) Development of the myotomal neuromuscular junction in Xenopus laevis: an electrophysiological and fine-structural study. Dev Biol 60:101-129.

Liley AW (1956) An investigation of spontaneous activity at the neuromuscular junction of the rat. J Physiol (Lond) 132:650-666.

Love FM, Thompson WJ (1996) Rat neuromuscular junctions gain terminal Schwann cells during postnatal development. Soc Neurosci Abstr 22:1474.

Morrissey TK, Levi ADO, Nuijens A, Sliwkowski MX, Bunge RP (1995) Axon-induced mitogenesis of human Schwann cells involves heregulin and p185erbB2. Proc Natl Acad Sci USA 92:1431-1435.
Noakes PG, Bennett MR (1987) Growth of axons into developing muscles of the chick forelimb is preceded by cells that stain with Schwann cell antibodies. J Comp Neurol 259:330-347.

Pellegrino RG, Spencer PS (1984) Schwann cell mitosis in response to regenerating peripheral axons in vivo. Brain Res 341:16-25.

Peters A, Muir AR (1959) The relationship between axons and Schwann cells during development of peripheral nerves in the rat. J Exp Physiol 44:117-130

Pomeroy SL, Purves D (1988) Neuron/glia relationships observed over intervals of several months in living mice. J Cell Biol 107:1167-1175.

Raabe TD, Clive DR, Neuberger TJ, Wen D, DeVries GH (1996) Cultured neonatal Schwann cells contain and secrete neuregulins. J Neurosci Res 46:263-270.

Reithmacher D, Sonnenberg-Reithmacher E, Brinkman V, Yamaai T, Lewins GR, Birchmeier C (1997) Severe neuropathies in mice with targeted mutations in the ErbB3 receptor. Nature 389:725-730.

Reynolds ML, Woolf CJ (1992) Terminal Schwann cells elaborate extensive processes following denervation of the motor endplate. J Neurocytol 21:50-66.

Robitaille R (1995) Purinergic receptors and their activation by endogenous purines at perisynaptic glial cells of the frog neuromuscular junction. J Neurosci 15:7121-7131.

Robitaille R, Jahromi BS, Charlton MP (1997) Muscarinic calcium responses resistant to muscarinic antagonists at perisynaptic Schwann cells of the frog neuromuscular junction. J Physiol (Lond) 504:337-347.

Romine JS, Bray GM, Aguayo AJ (1976) Schwann cell multiplication after crush injury of unmyelinated fibers. Arch Neurol 33:49-54.

Salzer JL, Bunge RP (1980) Studies of Schwann cell proliferation: I. An analysis in tissue culture of proliferation during development, Wallerian degeneration and direct injury. J Cell Biol 84:739-752.

Salzer JL, Bunge RP, Glazer L (1980a) Studies of Schwann cell proliferation: III. Evidence for the surface localization of the neurite mitogen. J Cell Biol 84:767-778.

Salzer JL, Williams AK, Glazer L, Bunge RP (1980b) Studies of Schwann cell proliferation: II. An analysis in tissue culture of proliferation during development, Wallerian degeneration and direct injury. J Cell Biol 84:739-752.

Scherer SS, Salzer JL (1996) Axon-Schwann cell interactions during peripheral nerve degeneration and regeneration. In: Glial cell development (Jessen KR, Richardson WD, eds), pp 165-196. Oxford: BIOS.

Slater C (1982) Postnatal maturation of nerve-muscle junctions in hindlimb muscles of the mouse. Dev Biol 94:11-22.

Son Y-J, Thompson WJ (1995a) Schwann cell processes guide regeneration of peripheral axons. Neuron 14:125-132.

Son Y-J, Thompson WJ (1995b) Nerve sprouting in muscle is induced and guided by processes extended by Schwann cells. Neuron 14:133-141.

Speidel CC (1964) In vivo studies of myelinated nerve fibers. Int Rev Cytol 16:173-231.

Swartz DJ, Santi PA (1996) Immunofluorescent artifacts due to the $\mathrm{pH}$ of anti-fading mounting media. Biotechniques 20:398-400.

Thompson WJ, Jansen JKS (1977) The extent of sprouting of remaining motor units in partly denervated immature and adult rat soleus muscle. Neuroscience 2:523-535.

Trachtenberg JT, Thompson WJ (1996) Schwann cell apoptosis at developing neuromuscular junctions is regulated by glial growth factor Nature 379:174-177.

Trachtenberg JT, Thompson WJ (1997) Nerve terminal withdrawal from rat neuromuscular junctions induced by neuregulin and Schwann cells. J Neurosci 17:6243-6255.

Usson Y, Saxod R (1988) Schwann cell proliferation in the sciatic nerve of hypothyroid chick embryos studied by autoradiography and image analysis. J Neurocytol 17:639-648.

Waerhaug O (1992) Postnatal development of rat motor nerve terminals. Anat Embryol 185:115-123.

Wood PM, Bunge RP (1975) Evidence that sensory axons are mitogenic for Schwann cells. Nature 256:662-664.

Yntema CL (1943) Deficient efferent innervation of the extremities following removal of neural crest in Amblystoma. J Exp Zool 94:319-349. 